\title{
A Genetic Algorithm for Mid-Air Target Interception
}

\author{
Irfan Younas \\ HITEC University Taxila cantt. \\ Pakistan
}

\author{
Atif Aqeel \\ PMAS-AAUR Rawalpindi \\ Pakistan
}

\begin{abstract}
This paper presents a computational model that uses a modified Genetic Algorithm (GA) approach, to provide more accurate results for mid-air targets interception. The proportional navigation laws that have been practiced for many years for target interception have been deployed in this research. A revised GA is formulated to determine the optimal interception point by modifying the heuristic crossover. An interception point is computed in which the miss distance and missile flight times are minimized. The selections of proportional navigation constant $\left(\mathrm{C}_{\mathrm{N}}\right)$ and time-to-launch values are playing a key role in minimizing the interception error. The results suggest a minimized interception error with better accuracy for target interception.
\end{abstract}

\section{General Terms}

Missile Dynamics and Evolutionary Algorithms.

\section{Keywords}

Genetic algorithms, Target interception and Missile dynamics.

\section{INTRODUCTION}

Genetic Algorithms have been applied in various fields to find out a near optimal solution in a relatively short period of time. The mid-air target interception is a tactical problem, where accuracy of system is paramount to intercept the required target within short, measured time bounds. Some recent works include artificial intelligence approaches to derive an accurate solution, but lack of required accuracy is still causing great difficulties. Miss distance and flight time are two factors that play a critical role and should be minimized to determine an optimal solution.

Proportional navigation laws have been implemented in missile target interception which provides reasonably good results [1]. The value of navigation constant $\left(\mathrm{C}_{\mathrm{N}}\right)$ is important to improve the performance of system. The closed-form approach has been used for non-maneuvering targets under the proportional navigation laws [2], but there is a need to provide an approach that is effective for intercepting maneuvering targets. Theories for target tracking and guidance laws have been revised time to time [3 5]. However, most of these approaches are designed to solve the target interception problem in two dimensional systems only. Furthermore, these approaches have to fulfill certain conditions and to work in specific environment. Hence, a real-time scenario is hard to model due to application of these conditions. Also there is a need for high performance systems with very low computing time. Use of high end computing systems increases the costs, and limits the operating environment. Deployments of intelligent approaches are helpful to overcome cost constraints and higher accuracy can also be achieved.

Some recent research includes intelligent systems which has promising results. Here Genetic Algorithms (GAs) have been used for path optimization of multiple missiles having same origin and common interception point [6]. A neural network was implemented for ballistic missile to intercept a non-maneuvering target, where three-dimensional trajectory data was used for training of the neural network based guidance system [7]. In another instance an enforced sub-population evolutionary algorithm was used to design a rocket guidance system, again a neural network system was trained through algorithm results [8]. The use of GA as optimization tool for handling the angle of attack for missile flight trajectory was deployed in [9]. In order to minimize miss distance and flight time, an effective algorithm was proposed for non-maneuvering targets interception, which also contained an embedded neural network to increase the system performance $[10,11]$.

In this research, a modified GA approach is proposed, which uses heuristic crossover as the greedy approach to provide a near optimal solution for target interception. The inputs to the modified GA are target and missile dynamics, which incorporates the proportional navigation law, while the outputs of the GA are optimized values of the navigation constant $\left(\mathrm{C}_{\mathrm{N}}\right)$ and missile time-to-launch. A fitness function is constructed to minimize the flight time and miss distance leading to target interception. The obtained results are promising and indicative of capabilities of the new system.

\section{MID-AIR TARGET INTERCEPTION}

Target interception can generally be classified as a non-linear problem, which should be able to handle the dynamics of missile and targets in an efficient way. External factors such as air and turning rate can affect the motion of missile and that should form part of the maneuvering dynamics. The computation has been made with respect to a three dimensional coordinates system. The efficiency of the system is naturally dependent on computation time requirements. Hence in the proposed algorithm a greedy GA approach is deployed so that minimum time should be consumed to find an optimal solution.

The proportional navigation laws are deployed during the presentation of dynamics. The genetic algorithm has embedded the heuristic crossover approach which is to minimize the missile flight time ( $\left.\mathrm{F}_{\text {missile }}\right)$ and target miss-distance $\left(\mathrm{D}_{\text {miss }}\right)$. In 
constructing the fitness function the aim is to minimize the above two parameters. The adjustment of value for the navigation constant $\left(\mathrm{C}_{\mathrm{N}}\right)$ can provide us with minimized flight time, so it must be determined. The missile time-to-launch (M-launch) will be computed according to the target position. M-launch will be computed in order to minimize the flight time.

The research work is divided into three sections, where the first and second section contains dynamics of missile and target, while third section provides details of the proposed genetic algorithm and the data to be used as input.

\subsection{Target Dynamics Modeling:}

Figure 1 describes the overall scenario; it shows a guarding or defending zone within a three dimensional coordinates system. It further displays the boundary of defending zone, having missile launch point situated at its origin while a target can enter from any point. The maximum and minimum altitudes are defined by the $\mathrm{z}$-axis, and the negative $\mathrm{z}$-axis is ignored.

In agreement with the proportional navigation law, a line of sight angle will be maintained as soon as target enters the defending zone. This defending zone is calculated and fixed depending on the missile range. The intruding object, also termed as the target will have it dynamics recorded as it enters the defending zone mentioned above.

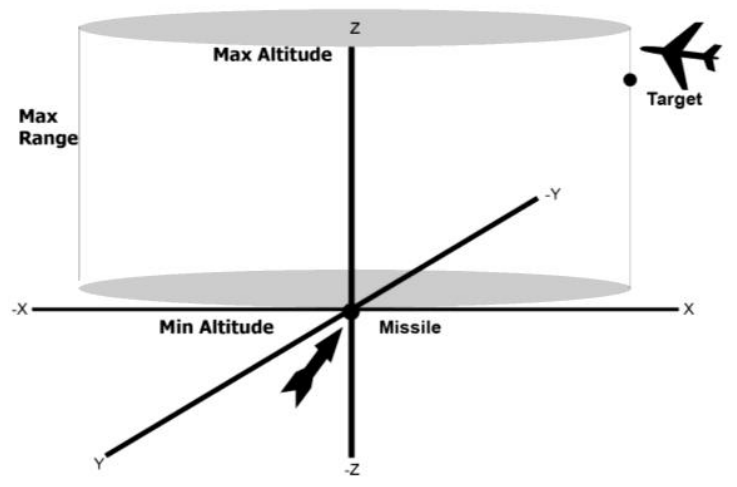

Figure-1: Defending Zone

The target dynamics are extracted according to the motion laws [11], where the initial tracking data that is required for computation are the target initial position $(\mathrm{x}, \mathrm{y}, \mathrm{z})$, its velocity $(\mathrm{V}$ target), and angles according to $\mathrm{x}$ - and $\mathrm{y}$-axes.

The target motion path points are calculated using the equations below.

$$
\begin{aligned}
& \mathrm{X}_{\text {target }}(\mathrm{t})=\mathrm{X}_{\text {target }}(\mathrm{t}-1)+\mathrm{V}_{\text {target }}(\mathrm{t}) \cos \theta_{1}(\mathrm{t}) \cos \theta_{2}(\mathrm{t}) \\
& \mathrm{Y}_{\text {target }}(\mathrm{t})=\mathrm{Y}_{\text {target }}(\mathrm{t}-1)+\mathrm{V}_{\text {target }}(\mathrm{t}) \sin \theta_{1}(\mathrm{t}) \cos \theta_{2}(\mathrm{t}) \\
& \mathrm{Z}_{\text {target }}(\mathrm{t})=\mathrm{Z}_{\text {target }}(\mathrm{t}-1)+\mathrm{V}_{\text {target }}(\mathrm{t}) \sin \theta_{2}(\mathrm{t})
\end{aligned}
$$

Where " $t$ " is the time, $\theta_{1}$ is the angle of velocity projected on to $\mathrm{x}$-axis, while $\theta_{2}$ is the angle of velocity with respect to $\mathrm{y}$-axis.

The change in velocity $\mathrm{V}_{\text {target }}$ and the change in angles $\theta_{1}$ and $\theta_{2}$ can be computed using equations $2.4,2.5$ and 2.6 respectively.

$$
\begin{aligned}
& \mathrm{V}_{\text {target }}(\mathrm{t})=\mathrm{V}_{\text {target }}(\mathrm{t}-1)+\mathrm{st} \Delta \mathrm{V}(\mathrm{t}) \\
& \theta_{1}(\mathrm{t})=\theta_{1}(\mathrm{t}-1)+\mathrm{st} \Delta \theta_{1}(\mathrm{t}) \\
& \theta_{2}(\mathrm{t})=\theta_{2}(\mathrm{t}-1)+\mathrm{st} \Delta \theta_{2}(\mathrm{t})
\end{aligned}
$$

Where "st" indicates the sampling time, which is the time consumed to track the position of target. The $\Delta$ shows the change in parameters values.

Figure-2 illustrates the dynamics of target with respect to the coordinates system as it enters point of defending zone.

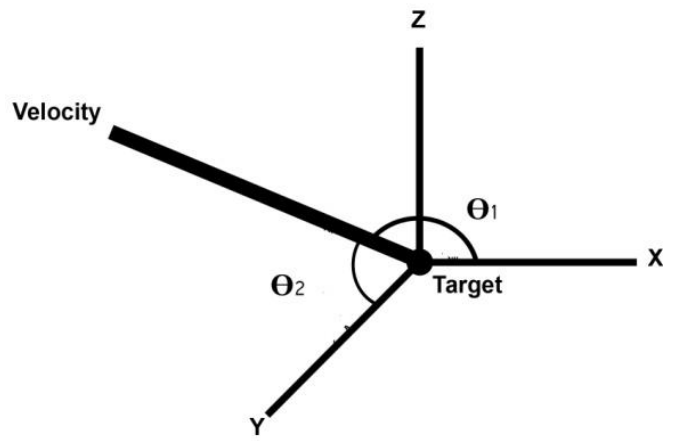

Figure-2: Target Dynamics

The target motion path is computed through equations (2.1) to (2.6), this path describes the entrance and exit points of the target in the defending zone.

Equation (2.7) provides the range of target within the defending zone at any time ' $\mathrm{t}$ '.

$$
\mathrm{R}_{\text {target }}(\mathrm{t})=\sqrt{\left(\mathrm{X}_{\text {target }}(\mathrm{t})\right)^{2}+\left(\mathrm{Y}_{\text {target }}(\mathrm{t})\right)^{2}+\left(\mathrm{Z}_{\text {target }}(\mathrm{t})\right)^{2}}
$$

\subsection{Missile Dynamics Modeling:}

As shown in figure-1, the missile launch point is placed at the origin of defending zone. The dynamics of missile should include the missile motion, acceleration, and its thrust power. In accordance with the proportional navigation law, the line of sight angles should be used to find a target interception point. Therefore the extraction of missile acceleration commands to intercept the required target [12], equations (2.8) to (2.11) will be used to compute the velocity of missile $\left(\mathrm{V}_{\text {missile }}\right)$ and angles of acceleration. 


$$
\mathrm{V}_{\text {missile }}(\mathrm{t})=\mathrm{V}_{\text {missile }}(\mathrm{t}-1)+\operatorname{st~Ac}_{\text {missile }}(\mathrm{t})
$$

Where "Ac missile" is actual acceleration of missile and "st" is sampling time for measuring the acceleration.

$$
\mathrm{A}_{1 \text { missile }}(\mathrm{t})=\frac{\text { (Thrust } \left._{\text {target }}+\text { Drag }_{\text {target }}\right)}{\text { Mass }_{\text {target }}}
$$

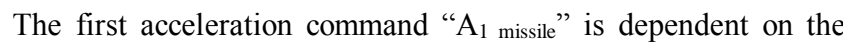
values of thrust, drag and mass values of target. This acceleration command works parallel to the velocity of missile $\left(\mathrm{V}_{\text {missile }}\right)$.

$\mathrm{A}_{2 \text { missile }}(\mathrm{t})=\mathrm{C}_{\mathrm{N}} \Phi_{1}(\mathrm{t})$

$\mathrm{A}_{3 \text { missile }}(\mathrm{t})=\mathrm{C}_{\mathrm{N}} \Phi_{2}(\mathrm{t})$

"A 2 missile" and "A 3 missile" are the second and third acceleration commands which work perpendicular to the missile velocity ( $\mathrm{V}$ missile). Here " $\mathrm{C}_{\mathrm{N}}$ " is the proportional navigation constant, which value needs to be examined for accurate target interception, while " $\Phi_{1}$ " and " $\Phi_{2}$ " are the projection angles of line of sight with respect to $\mathrm{x}$ - and $\mathrm{y}$-axis.

It should be noted here that since the Proportional Navigation law dictates that two vehicles are on a collision course when their direct Line-of-Sight (LOS) does not change direction. Hence the missile velocity vector should rotate at a rate proportional to the rotation rate of the LOS-rate, and in the same direction. This will give rise to acceleration components perpendicular to the missile velocity.

Figure-3 shows the details of angles, velocity and the acceleration commands of missile with respect to the origin point. The missile line of sight angle should be maintained with respect to the motion of target, according to the proportional navigation law and the navigation constant can have constant value or it may vary according to situation. In our scenario, its value will vary. The value will be chosen such that it is best fitted in given scenario, providing minimized miss distance and flight time after optimization.

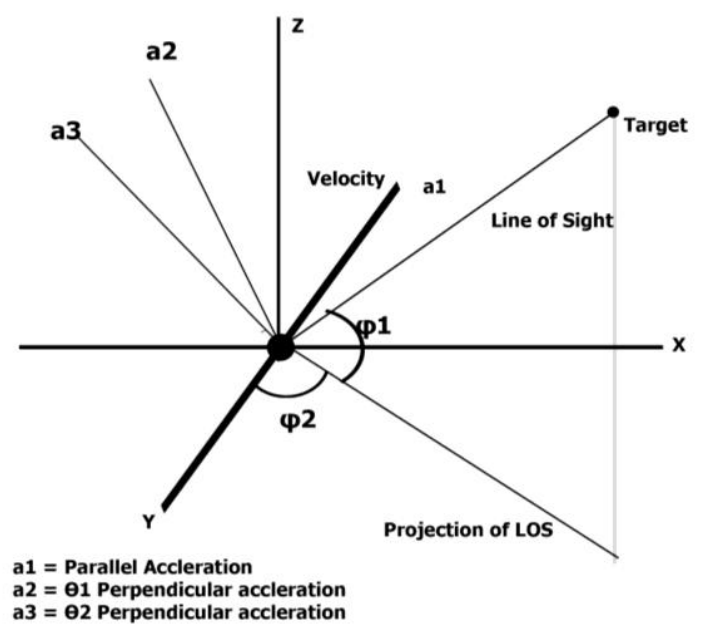

Figure-3: Missile Dynamics

\subsection{Modified Genetic Algorithm:}

In order to find an optimized solution to the problem, within a very short period of time the use of Genetic Algorithms (GAs) is one of the best approaches that can provide a near optimal solution. For the GA to compute an optimized solution to a certain level there is a need to modify the algorithm to get better results. Due to the non-linearity of target interception problem, a simple genetic algorithm can optimize values, but the interception error rate is too high. Therefore a heuristic crossover approach is embedded in our proposed solution having the following steps.

i. Generate the initial population of individuals using the uniform function. During this $M$ individuals will be created.

ii. Calculation of fitness of individuals' will be done through a fitness function.

iii. To select individuals for reproduction or mating half of the individuals will be selected using roulette selection.

iv. The reproduction will be done by exchanging the offspring of two individuals with a fraction rate of 0.8 . While the crossover will be performed using heuristic function with a ratio of 1.2.

v. The mutation operation will be applied for more accuracy, and uniform mutation will be applied over new individuals with a ratio of 0.01 .

vi. Repeat the steps from (ii) to (v) to obtain a best value.

The above steps are repeated for a number of generations which will provide us optimal value of navigation constant $\left(\mathrm{C}_{\mathrm{N}}\right)$ and the missile time-to-launch according to the computations. The fitness function for minimizing the miss distance and flight time is given in equation (2.12).

$$
\mathrm{K}=\min \left\{\sqrt{\left(\mathrm{W}_{1} \mathrm{~F}_{\text {missile }}\right)^{2}+\left(\mathrm{W}_{2} \mathrm{D}_{\text {miss }}\right)^{2}}\right\}
$$

Where $\mathrm{W}_{1}$ and $\mathrm{W}_{2}$ are the weights to balance the values obtained through optimization. The minimum value of " $\mathrm{K}$ " is considered as end of iteration according to the step (vi) of the proposed algorithm.

In optimization of miss distance and flight time, the target dynamics are the population for genetic algorithm. Where the whole path points obtained using equations (2.1) to (2.6) are the population. A chromosome of population is the set of nine values as genes which includes $\mathrm{X}_{\text {target, }}, \mathrm{Y}_{\text {target, }} \mathrm{Z}_{\text {target }}, \mathrm{V}$ target, $\theta_{1}, \theta_{2}$, $\Delta \mathrm{V}$ target, $\Delta \theta_{1}, \Delta \theta_{2}$. The real format values of these variables are used for optimization. Total $\mathrm{M}$ number of population take part as input to modified genetic algorithm. The computation of fitness function is calculated by giving different values of navigation constant $\left(\mathrm{C}_{\mathrm{N}}\right)$ which varies from 1 to 50 . The missile dynamics is computed by deploying equations (2.8) to (2.11).

The reproduction of new off springs from $\mathrm{M} / 2$ selected chromosomes is done using heuristic crossover. Two fitted chromosomes are taken and mated according to heuristic 
crossover to produce two new off springs. The heuristic crossover is illustrated as follows.

Offspring-1 = BestParent $+\mathrm{r}^{*}($ BestParent - WorstParent $)$ (2.13)

Offspring-2 $=$ BestParent

One of the offspring is created using the best parent (chromosome) while the second offspring is based over the formula in equation (2.14). The value of $\mathrm{r}$ varies from 0 to 1 . After a number of defined iteration, if some solution is not found for Offspring-1 then worst parent is taken as Offspring-1. After reproduction of new off springs, we have again $M$ populations that have more fitted chromosomes as compared to the initial population. This heuristic crossover is termed a greedy approach in this research, and if it finds a best value early then it moves there, which on the whole can save a lot of critical computation time.

The time target takes to reach boundary of defending zone, the maximum time to stay in range, minimum and maximum altitude is calculated using the following equations.

$$
\begin{aligned}
& \mathrm{R}_{\text {max }}=-2\left(\mathrm{Z}_{\text {target }} \sin \left(\theta_{2}\right)+\mathrm{X}_{\text {target }} \cos \left(\theta_{1}\right) \cos \left(\theta_{2}\right)\right. \\
& +\mathrm{Y}_{\text {target }} \sin \left(\theta_{1}\right) \cos \left(\theta_{2}\right) / \mathrm{V}_{\text {target }}
\end{aligned}
$$

Alt $\max =\frac{\left(Z_{\text {max }}-Z_{\text {target }}\right)}{V_{\text {target }} \sin \left(\theta_{2}\right)}$

$A l t_{\text {min }}=\frac{\left(Z_{\text {min }}-Z_{\text {target }}\right)}{V_{\text {target }} \sin \left(\theta_{2}\right)}$

Where $\mathrm{Alt}_{\max }$ and $\mathrm{Alt}_{\min }$ are maximum altitude and minimum altitude respectively, while the $Z_{\max }$ and $Z_{\min }$ are the values of maximum and minimum of the $\mathrm{z}$-axis.

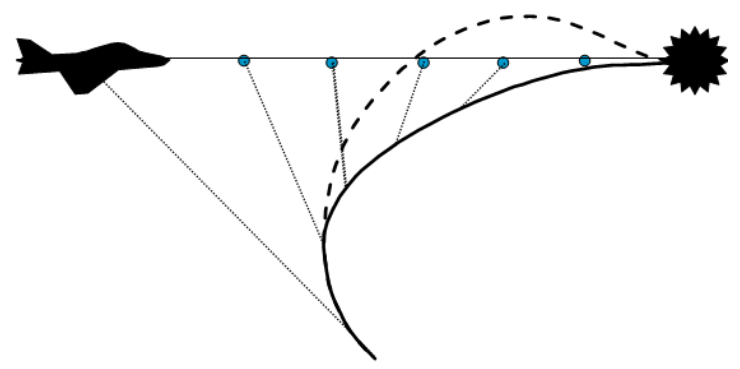

Figure-4: Pursuit path with line of sight [13]

There are some conditions that have to be met by this overall scenario. First, the missile owns flight time should be greater than the computed time flight time. Second, for computation purposes, the target should be within the defending zone range and altitudes. Third, entry point of target in defending zone that point is to be taken as initial point to perform computations and optimization.

The optimization with respect to the proportional navigation laws will follow the above scenario as illustrated in figure-4 [12]. The line of sight will be maintained to calculate an interception point, and for that interception point, a GA is optimizing the values of miss distance and flight time of missile.

The miss distance and flight time is calculated according to the scenario derived from examining points of target motion, and where a less miss distance and flight time is involved, genetic algorithm will take it as optimal point, and last point is replaced by this one. This scenario is depicted in figure- 5 .

The results of optimization is providing values for the navigation constant that is best fitted according to the scenario and also the missile time-to-launch depending on selected interception point.

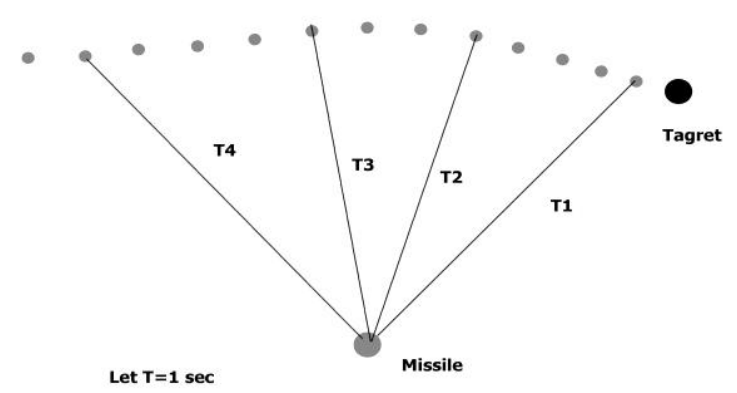

Figure-5: Miss Distance Scenario

\section{RESULTS}

A scenario for the missile target interception is constructed with values that are gained from real environment. It was assumed that target is entering in defending zone with initial position Pos $(\mathrm{X}=3780, \mathrm{Y}=13860, \mathrm{Z}=4314)$ with respect to the origin. The origin of the defending zone is the point from where a missile is to be launched. The target velocity $\mathrm{V}_{\text {target }}$ is $220 \mathrm{miles} / \mathrm{second}$, while the angle $\theta_{1}$ is $184^{\circ}$ and the $\theta_{2}$ is $72^{\circ}$. The scan time for radar is 0.05 second, while the maximum flight time for missile is given as $\mathrm{MF}_{\max }=50$ seconds. The target thrust, drag and mass are taken $220 \mathrm{~m} / \mathrm{s}^{2}, 0.000444$ and $10000 \mathrm{~kg}$ respectively.

The change in the velocity and the angles of the radar was then updated according to the scan time. The following table (Table 1) gives the target initial change velocity and angles.

Table 1: Change in target Motion

\begin{tabular}{|l|l|l|l|}
\hline $\begin{array}{l}\text { St } \\
\text { (sampling time) }\end{array}$ & $\boldsymbol{\Delta V}$ target & $\Delta \boldsymbol{\theta}_{1}$ & $\Delta \boldsymbol{\theta}_{\mathbf{2}}$ \\
\hline 0.05 & $4 \mathrm{~m} / \mathrm{sec}$ & $4^{\circ}$ & $2^{\circ}$ \\
\hline
\end{tabular}

The computation results show, target will remains within defending zone for 127.21 seconds according to equation 2.15, while the calculated value of maximum altitude using equation 2.16 is -31.48 , where the negative sign indicates that target is 
unable to gain maximum altitude. The equation 2.17 provides a time for reaching the minimum altitude which is 27.12 seconds

The next step of the proposed approach is to determine the position of the target using the change in velocity and angles. The change in position along with angles provides the path of the target in the defending zone. The missile trajectory and point of interception depends on the scan time along with the path of target. Furthermore, computations are made using the target dynamics equations 2.1 to 2.6 to gain the point of interception with the missile dimensions along with selection of value for navigation constant. The scan time is taken as 0.05 second, while the maximum missile flight time is 50 seconds.

A population of total 2540 chromosomes were generated to find an optimized solution. The optimization results obtained through simulation contains: the best miss distance of $1.56 \mathrm{~m}$ corresponding to flight time of 49 seconds; the navigation constant value which ranges from 1 to 50, obtained through GA optimization is 49 and the time to launch for missile is 77 seconds after the target enters into defending zone. These results are as shown in table 2 .

Table 2: Optimization results

\begin{tabular}{|l|l|l|l|}
\hline $\begin{array}{l}\mathbf{C}_{\mathbf{N}} \\
\text { (Navigation } \\
\text { constant) }\end{array}$ & $\begin{array}{l}\mathbf{T}_{\text {launch }} \\
\text { (Time } \\
\text { launch) }\end{array}$ & $\begin{array}{l}\text { Dm } \\
\text { (Miss } \\
\text { distance) }\end{array}$ & $\begin{array}{l}\mathbf{F}_{\text {missile }} \\
\text { (Missile } \\
\text { flight time) }\end{array}$ \\
\hline 49 & $77 \mathrm{sec}$ & $1.56 \mathrm{~m}$ & $49 \mathrm{sec}$ \\
\hline
\end{tabular}

\section{CONCLUSIONS}

This paper has presented a modified genetic algorithm that provides near optimal results for the mid-air target interception in the three-dimensional coordinate system. The results shown miss distance error of $1.56 \mathrm{~m}$, which needs some improvements, but that does not mean, this approach provides same error rate for all situations. The miss distance error can be minimized for maneuvering targets but it cannot be zero. The future work will include the neural network based approach for mid-air target interception, which will increase the performance of overall system through faster computations.

\section{REFERENCES}

[1] P.R Mahapatra and U. S. Shuk (1989), Indian Institute of Science, "Accurate Solution of Proportion Navigation for Maneuvering Targets",IEEE Transactions On Aerospace And Electronic Systems Vol. Aes-25.

[2] K. Becker (1990), "Closed-form solution of pure proportional navigation," IEEE Transactions on Aerospace and Electronic Systems, vol. 26, pp. 526-533.
[3] P. Zarchan (1999), "Ballistic Missile Defense Guidance and Control Issues," SCIENCE AND GLOBAL SECURITY, vol. 8, pp. 99-124.

[4] D. MacKenzie,(1988) The Soviet Union and Strategic Missile Guidance, International Security, vol. 13, pp. 5-54.

[5] Dyer, W. R. (2004). Modern Missile Analysis Guidance, Control, Seekers, and Technology, U.S. Department of Defense, www.aticourse.com, Slides 1-35.

[6] Hughes, E. J. (2002). Evolutionary guidance for multiple missiles, 15th Triennial World Congress, Barcelona, Spain, IFAC, pp. (no-paging).

[7] Song, E. J. (2002). Three-dimensional midcourse guidance using neural networks for interception of ballistic targets, IEEE Transactions on Aerospace and Election Systems, Vol. 38, No. 2, pp. 404-414.

[8] Gomez, F. J. and R. Miikkulainen. (2003). Active guidance for a finless rocket using neuroevolution, Genetic Evolutionary Computation Conference 2003, GECCO Neural Network Research Group, (http://nn.cs.utexas.edu), 7 pp. (no paging).

[9] Yang, Z. H., J. C. Frang and Z. Q. Qi. (2005). Flight midcourse guidance control based on genetic algorithm, GECCO 05, Washington, DC, USA, ACM, pp. 1501-1506.

[10] Madkour, A. A. M., M. A. Hossain and K. P. Dahal. (2005). Evolutionary missile guidance algorithms for interception of maneuvering targets in 3D environment, IEEE, $4 \mathrm{pp}$. (no paging). [11] Madkour, A. A. M., M. A. Hossain and K. P. Dahal. (2006). Evolutionary optimization approach for missile guidance algorithms to achieve accurate interception of maneuvering targets in 3D, IEEE Control, Automation and Signals Processing Professional Network, pp. 101-104.

[12] L. Lin, T. Kirubarajan, and Y. Bar-Shalom, (2005). Pursuer identification and time-to-go estimation using passive measurements from an evader, IEEE Transactions on, Aerospace and Electronic Systems vol. 41, pp. 190 - 204.

[13] Szypula, (2005). Guidance and control principles. US Navall Weapons Systems, http://www.famu.edu/ Guidance_and_Control_Principles.PPT, Slides 1-20. 